\title{
How to maintain a marriage: Maintenance behaviors, equity, and appreciation in understanding marital satisfaction ${ }^{+}$
}

\author{
Nazlı Büşra Akçabozan Kayabol ${ }^{1}$ (D) $\cdot$ Zeynep Hatipoğlu Sümer ${ }^{2}$
}

(C) Springer Science+Business Media, LLC, part of Springer Nature 2020

\begin{abstract}
This study investigated the role of self-reported and perceived partners' use of maintenance behaviors (openness, sharing tasks, and positivity) on marital satisfaction through the indirect roles of relational equity and appreciation (felt and expressed). We used a sample of 602 married individuals living in Turkey to test two hypothesized models using Structural Equation Modeling. Our results from the first model indicated that the indirect associations between self-reported use of maintenance behaviors and marital satisfaction were explained by the individual mediator role of felt appreciation and serial indirect role of felt appreciation and relational equity. On the other hand, the results of the second model indicated that the indirect associations between partners' use of maintenance behaviors and marital satisfaction were explained by the individual mediator roles of relational equity and expressed appreciation as well as the serial indirect role of relational equity and expressed appreciation. We discussed the implications for theory, research and practice, and recommendations for further studies.
\end{abstract}

Keywords Appreciation $\cdot$ Equity theory $\cdot$ Marital satisfaction $\cdot$ Relationship maintenance $\cdot$ Structural equation modeling

\section{Background}

Marital satisfaction (MS) can be briefly described as an individual's "emotional state of being content with the interactions, experiences, and expectations of his or her married life" (Ward et al. 2009, p. 415). MS has received widespread attention in marital research. An empirical inquiry has revealed numerous determinants of MS to differentiate satisfying and unsatisfying marriages (Fincham et al. 2018) and considerable attention has been devoted to investigating relationship maintenance behaviors with the underlying assumption that satisfying relationships are not self-maintaining but require partner efforts (Canary and Stafford 1994; Duck 1988). Individuals

This study is part of the first author's Ph.D dissertation submitted to the Department of Educational Sciences, Middle East Technical University under the supervision of the second author

Nazlı Büşra Akçabozan Kayabol

busra.akcabozan@hku.edu.tr

Zeynep Hatipoğlu Sümer

zeynep@metu.edu.tr

1 Department of Educational Sciences, Hasan Kalyoncu University, 27100 Gaziantep, Turkey

2 Department of Educational Sciences, Middle East Technical University, 06800 Ankara, Turkey engage in various kinds of maintenance activities with the motives of mitigating threats and enhancing their relationships (Ogolsky et al. 2017). Across prior studies, researchers have made several conceptual clarifications and developed different typologies to categorize relationship maintenance activities and behaviors. In the present study, we focused on the most recognized relationship maintenance behaviors typology in the literature which was first developed by Stafford and Canary (1991) and which they advanced over the years (Canary and Stafford 1992; Stafford et al. 2000; Stafford 2011) intending to understand the impact of engagement in maintenance behaviors on promoting positive relationship processes such as satisfaction, commitment, liking, and love. Studies of this typology have been based on various theoretical frameworks, particularly from the perspective of equity theory (Hatfield et al. 1985) which claims that partners exchange a variety of rewards and costs in their relationships and perceive equity when the ratio of rewards to costs is proportionate (Hatfield and Rapson 2011; Sprecher and Schwartz 1994).

In the implementation of equity theory on relationship maintenance research, one's use of maintenance behaviors is considered to be his/her inputs (costs) for self; on the other hand, these inputs become outcomes (rewards) for the other partner (Canary and Stafford 1992; Stafford 2003). Empirical evidence has demonstrated that perceived relational equity is a 
predictor of engagement in self-reported and perceived partner use of maintenance behaviors (e.g., Canary and Stafford 1992; Dainton 2016; Jackson 2010). Along with its predictor role, relational equity as a desired state of marriage is also considered to be encouraged using maintenance behaviors (Dainton and Zelley 2006). In other words, relational equity is considered both as a predictor and an outcome of engagement in maintenance behaviors. Prior research has mostly investigated the predictor role of relational equity in explaining maintenance behaviors (e.g., Canary and Stafford 1992; Dainton 2016; Yum and Canary 2009); however, less is known about the role of relational equity as an outcome of maintenance behavior engagement. Inspired by this scarcity in the literature, we sought to provide a unique perspective of the bidirectional nature of the association between perceived equity and relationship maintenance behaviors. For this purpose, we both explored the role of relational equity as an outcome of selfreported and partner use of maintenance behaviors and as a mediator in understanding the interrelations between the use of maintenance behaviors (self and partner) and MS.

Based on the premises of equity theory, individuals in romantic relationships may experience imbalances in their sense of equity when they engage in more maintenance behaviors compared to their partners, which in turn affect relationship satisfaction negatively. It is here that another concept - appreciation - comes into prominence as having the ability to intervene in the negative association between perceived inequity and relationship satisfaction (Berger and Janoff-Bulman 2006). Despite the recent focus in the literature on the exploration of appreciation within the context of marital relationships, consistent evidence has affirmed the protective role of appreciation both for the recipients (felt appreciation) and expressers (expressed appreciation). Further, appreciation has been demonstrated as effective in promoting relationship maintenance as well as satisfaction (Algoe et al. 2010; Gordon et al. 2012; Joel et al. 2013; Lambert and Fincham 2011). Because self-reported engagement in maintenance behaviors could be perceived as costly by individuals despite the benefits (Stafford, 2003), they would want to be on the receiving end of appreciation. On the other hand, individuals who notice their partners' use of maintenance behaviors (rewards for themselves) may develop appreciative feelings as a response to this received benefit.

Furthermore, the limited existing studies (mostly conducted with wife-only samples, Hawkins et al. 1998) revealed that individuals who feel appreciated by their partners are more likely to perceive their relationships as equitable. In these cases, appreciation had an influential role in explaining the direct relationship between perceived inequity which results in engagement of costly behaviors (e.g., task sharing, household labor, and sacrifices) and relationship satisfaction (e.g., Berger and Janoff-Bulman 2006; Hawkins et al. 1998). Although the available literature has not yet provided evidence for the impact of expressed appreciation on the sense of equity, the potential for direct links between the given variables inspired us to examine the mediator role of felt and expressed appreciation with perceived relational equity to develop a clear understanding about the underlying associations between maintenance behaviors (self and partner use) and MS.

\section{Maintenance Behaviors}

Maintained relationships are defined as "relationships that are beyond the initiation stage and have not reached a dissolution phase" (Dainton 1994, p.1). Relationship maintenance is a broadly used term including a variety of activities that partners use to keep their relationships in a satisfactory state. Researchers have aggregated these activities into various typologies, and Stafford and Canary's (1991) original fivefactor typology (positivity, assurances, sharing tasks, openness, and social network) has become the frequently used model today. Canary and Stafford (1994) proposed that relationships would fall apart without the use of maintenance behaviors which are described as "actions and activities used to sustain desired relational definitions" (p. 5). Despite extensive use of the five-factor typology in the relationship maintenance literature over the past 25 years, research has shown variability in maintenance behaviors (e.g., Canary et al. 1993; Stafford et al. 2000). Most recently, Stafford (2011) revised the current measurements of relationship maintenance and suggested a seven-factor typology including the maintenance behaviors of positivity (being cheerful, optimistic, and uncritical), understanding (feelings of being understood), assurances (giving messages to stress the endurance of the relationship), relationship talk (disclosing one's desires for the relationship), self-disclosure (sharing ideas with a partner), networks (activities done with or help sought from friends and family members), and tasks (performing responsibilities).

Relationship maintenance behaviors have been associated with various relational outcomes, and consistent empirical evidence has shown that both self-reported and partner use of maintenance behaviors promote relationship/marital satisfaction (e.g., Dainton et al. 1994; Dainton and Aylor 2002; Johnson 2009; Stafford and Canary 1991; Stafford et al. 2000). In the recent reviews of a vast literature on relationship maintenance (Ogolsky and Bowers 2013; Ogolsky et al. 2017), maintenance strategies were found to be significantly and positively correlated to relationship satisfaction with the largest effects found for positivity and assurances and more moderate effects found for openness, sharing tasks, and social networks. Moreover, partner use of maintenance behaviors was found to have a higher functional utility on satisfaction than self-reported use, which demonstrates the importance of examining both self-reported and perceived partner 
engagement in maintenance behaviors (e.g., Lee 2006; Ogolsky and Bowers 2013).

The positive impact of engagement in maintenance behaviors on satisfaction has also been provided in dating and married relationship samples from different nations and cultures (e.g., Ballard-Reisch et al. 1999; Yum and Li 2007). In Turkey, only one study has been identified in which the predictor role of maintenance behaviors on MS was examined; however, it was conducted in a small sample of 31 married individuals (Torun 2005). Results showed that self-reported use of positivity and partner use of assurances followed by social networks and sharing tasks were positive and significant predictors of MS; however, this study was conducted using a very small and homogenous sample without consideration of equity theory principles.

\section{Equity}

For several decades, equity theory which is a theoretical framework of social exchange theory has become quite successful in understanding intimate relationships. The underlying assumptions of equity theory are that individuals (1) tend to maximize rewards and minimize costs to themselves in their relationships and (2) are more likely to maintain relationships that they expect to be rewarding (Sprecher 1992; Thibaut and Kelley 1959). According to equity theory, partners evaluate their rewards (any potential resources that partners benefit from such as support, intimacy, and sex) and costs (any potential resources that partners contribute to such as kindness and money), and the extent to which the ratio of rewards to costs is perceived as being proportionate determines the level of perceived equity (Hatfield et al. 1985). Undoubtedly, partners who perceive their marriages as equitable are more likely to feel satisfied than those who perceive their marriages as inequitable (e.g., Jackson 2010; Stafford and Canary 2006; Weigel et al. 2006) regardless of whether they are under-benefited (partners give more, receive less) or over-benefited (partners give less, receive more) (Floyd and Wasner 1994).

The equity principle that guides Canary and Stafford's (1994) maintenance research is that "people are more motivated to maintain equitable relationships than inequitable relationships" (p. 7). Accordingly, research has largely supported that perceived equity is a notable and positive predictor of self-reported and partner use of maintenance behaviors (e.g., Canary and Stafford 1992; Jackson 2010) while inequitable relationships lead to less frequent self-reported and partner use of maintenance behaviors (Canary and Stafford 2001; Dainton 2016; Stafford 2003; Stafford and Canary 2006). Additionally, research showed that the most satisfying marriages are the most equitable ones and higher levels of perceived equity in marriage correlate with more frequent use of maintenance behaviors (Stafford and Canary 2006). To substantiate the existing literature in this field, researchers have also been encouraged to explore relational equity as a consequence of the maintenance behaviors (Stafford 2003). Theoretically, maintenance behaviors are used to sustain desired relational outcomes, one being relational equity along with satisfaction (Dainton and Zelley 2006). Hence, the associations between the use of maintenance behaviors and perceived equity are likely to be bidirectional: equity leads to the use of maintenance behaviors and the use of maintenance behaviors leads to perceived equity. Nevertheless, the literature still lacks sufficient studies investigating the predictive role of engagement in maintenance behaviors on perceived equity and this issue requires further investigation.

The concept of equity may not be as salient in cultures that are not similar to the United States and Western Europe, and cultural variations may alter how romantic partners assess relational equity and use of maintenance behaviors (e.g., Inglehart and Welzel 2005; Powell 2005). For instance, studies investigating the moderator role of culture from the perspective of cultural modernization theory (e.g., Yum et al. 2015; Yum and Canary 2009) have shown that people who hold self-expression and secular values (i.e., representing modern and post-modern lifestyles and being directed to Westernization and exchange orientation) adopt an equity approach and prefer to put effort towards maintaining their relationships. Accordingly, in these studies, equity predicted the use of maintenance behaviors in samples from the U.S, Spain, and Japan (in a limited way); yet, this was not true in samples from China, South Korea, and the Czech Republic (Yum and Canary 2009). Similarly, the curvilinear association between equity and maintenance strategies (highest association for equitably treated partners, followed by over-benefited partners, and followed by under-benefited partners) were found significant only for the romantic partners from the U.S but not for the partners from Malesia and Singapore (Yum et al. 2015). Results indicating that culture can alter the role of equity on relationship maintenance show that researchers need to limit generalizations about equity theory and maintenance behaviors to countries that do not share similar Western values. They should also continue investigating how equity theory principles apply to relationship maintenance in samples culturally dissimilar to the U.S. We could argue that unlike the U.S and Western Europe, individuals, especially older generations, in Turkey are more likely to hold more collectivist, and less self-expression and secular values which might have an impact on how they adopt equity perspective in their romantic relationship maintenance (Gurbuz et al. 2018; Kagitcibasi 1997). To our knowledge, however, no study in Turkish literature has yet examined the perspective of equity theory in romantic relationships. Furthermore, the role of perceived equity concerning MS and maintenance is still not clear. 


\section{Appreciation}

Appreciation (used interchangeably with the term gratitude, Gordon et al. 2012) is described as a feeling of being thankful for one person, circumstance, or event, and being aware of its value (Berger 2000). It occurs between partners with the positive aspects of their partners/relationships and as a response to the efforts they make for and care and concern they show to each other. Felt appreciation from a partner, showing appreciation, and verbal gratitude to a partner were all found to function as positive and protective factors of relationship satisfaction (Algoe et al. 2010; Barton et al. 2015; Lambert and Fincham 2011; Schramm et al. 2005). Moreover, the benefits of appreciation in romantic relationships were identified regardless of gender, relationship length (Kubacka et al. 2011), and nation (e.g., China; Bello et al. 2010).

Appreciation serves in a proactive relationship maintenance role itself and existing research provided evidence for the significant and positive associations between felt/ expressed appreciation and the use of various forms of maintenance behaviors (e.g., responsiveness, comfort in voicing concerns, intimate sacrifices) (Lambert and Fincham 2011; Gordon et al. 2012; Young and Curran 2016). Kubacka et al. (2011) investigated the dual function of gratitude and revealed that the spouse's gratitude was significantly associated with his/her use of maintenance behaviors and his/her spouse's gratitude. Additionally, the spouse's use of maintenance behaviors predicted his/her spouse's gratitude.

Feeling appreciated by a spouse was found to be a positive predictor of women's sense of fairness in the division of housework and child care (Hawkins et al. 1998) and women who felt more appreciated reported feeling more satisfied even when they engaged in more relational costs (Berger and Janoff-Bulman 2006). Consequently, felt appreciation appears to promote engagement in prosocial activities even if these activities would be costly and mitigate the sense of inequity. However, available research is limited in two ways (e.g., Berger and Janoff-Bulman 2006; Hawkins et al. 1998). First, equity has only been examined in terms of allocation of family work/household labor mostly in only-wife samples; and second, equity has been related to felt but not expressed appreciation. Nonetheless, we could argue that partners who express appreciation could benefit from doing so. Further, appreciativeness toward a partner would increase one's perception that the relationship is characterized by higher levels of perceived equity and satisfaction. It should also be noted that the unique role of each maintenance behavior (based on Stafford and Canary's typology) which pertains to appreciation has not yet been determined. Besides this, the existing literature on appreciation in Turkey is still limited to only a couple of studies in which either instrument was adapted to the Turkish language to gauge the concept of gratitude/ appreciation (e.g., Akın and Yalnız 2015) or appreciation was related to some concepts such as forgiveness and vengeance (Satıc1 et al. 2014).

Taken together, the facilitating roles of maintenance behaviors, equity, and appreciation have become an interest in studies examining MS; however, each construct has been investigated separately. Furthermore, to our knowledge, the indirect roles of appreciation and relational equity have not yet been examined. This study converges two areas of interest in relationship maintenance research; first, the importance of relational equity, and second, the felt and expressed appreciation in promoting satisfying marriages. Moreover, because the role of maintenance behaviors can vary depending on the type of behavior and on the reporter, we examined both self-reported and perceived partner use of each maintenance behavior. In light of the previous research to date and drawing upon the lacuna in the literature, we proposed that perceived equity and appreciation are factors that have indirect roles to explain the relationships between the use of maintenance behaviors and MS depending upon the reporter (individuals' own or partner's use of maintenance behaviors). Therefore, we tested two models and hypothesized that there will be a positive direct relationship between individuals' MS and selfreported use (Model 1, Hypothesis 1) and partner use of each maintenance behavior (positivity, sharing tasks, openness, Model 2, Hypothesis 2); MS and perceived equity (Model 1 and 2, Hypothesis 3); and MS and felt (Model 1, Hypothesis 4) and expressed appreciation (Model 2, Hypothesis 5).

We also predicted a positive relationship between perceived equity and felt (Model 1, Hypothesis 6) and expressed appreciation (Model 2, Hypothesis 7). Because less is known about the indirect roles of equity and appreciation (felt and expressed), we addressed two research questions. RQ1: How do felt appreciation and perceived equity indirectly (individually and in serial) relate to the role of each self-reported maintenance behavior on MS? RQ2: How do perceived equity and expressed appreciation indirectly (individually and in serial) relate to the role of self-reported or partner use of maintenance behaviors on MS?

\section{Method}

\section{Participants}

Our research sample included 602 heterosexual, married individuals ( 335 female, $55.6 \%$; 265 male, $44 \%$; 2 were undisclosed, $0.3 \%$ ) living in various cities in Turkey. A majority of participants were living in the capital city $(66.9 \%)$ and the remaining were from other metropolitan areas of Turkey. A total of $482(80.1 \%)$ individuals participated in the paperpencil survey, and 120 (19.9\%) individuals participated in the online survey. The age range for the sample $(n=589 ; 13$ were missing) was between 21 and 67 years $(M=38.47, S D=$ 
9.67). A majority of the participants $(n=259,43.0 \%)$ reported being university graduates while less than a quarter reported being high-school graduates $(n=137,22.8 \%)$, and the smallest group were masters or Ph.D. graduates $(n=92$, $15.3 \%)$. Most of the participants were in their first marriages $(n=570,94.7 \%)$. Marital length ranged from 1 year to 45 years $(M=11.92, S D=9.93)$. Majority of the participants $(n=443,73.6 \%)$ had children and the mean age of the firstborn was 12.83 years $(n=406, S D=9.95)$.

\section{Instruments}

\section{Demographic Information Form}

We developed a demographic information form to gather information about participants including their gender, age, level of education, and years married, etc.

\section{Relationship Maintenance Behaviors Measurement (RMBM)}

We used the revised version of the RMBM (Stafford 2011) to assess self-reported and perceived partner use of maintenance behaviors. RMBM includes seven factors ( 28 items; $1=$ strongly disagree to $7=$ strongly agree): positivity, understanding, self-disclosure, relationship-talks, assurances, tasks, and networks. Cronbach alpha values for each factor ranged from .82 to .95 . Higher scores indicate higher self-reported and partner use of maintenance behaviors. For the study, we instructed participants to assess both their self-reported and partner's use of each maintenance behavior consecutively. An adapted version of the RMBM (translated into Turkish) was examined in a sample of 418 married Turkish individuals (Akçabozan Kayabol 2017). According to the results of the Turkish factor structure of the RMBM, items clustered around five factors: openness ( 9 items, e.g., "I am open about my feelings"), positivity (10 items, e.g., "I act optimistically when I am with him/her"), sharing tasks (4 items, e.g., "I perform my household responsibilities"), social network of friends (2 items, e.g., "I do things with our friends"), and social network of family ( 2 items, e.g., "I ask a family member for help"). In the adaptation study, one item ("I spend time with our families") of the social network factor in the original study did not load on the social network of friends and social network of family factors and excluded from the scale. Therefore, the Turkish RMBM used in our study was comprised of 27 items. Cronbach's alpha values reflected the reliability of the factors (ranged from .79 to .94).

The factor structure of the Turkish RMBM resembles the previous categorization of maintenance behaviors in which positivity and openness factors were represented by one factor (e.g., Canary and Stafford 1992; Stafford and Canary 1991). Considering that "a factor with fewer than three items is generally weak and unstable" according to Costello and Osborne
(2005, p.5), we decided to exclude the social network of friends and social network of family factors in the test of structured models. In the current study, Cronbach's alpha values ranged between .82 to. 92 .

\section{Relational Equity Scale}

This scale was used to assess whether a respondent perceives that his/her relationship is equitable and whether s/he believes that s/he equally contributes to the relationship (Sabatelli and Cecil-Pigo 1985). The one-factor scale includes 10 items (e.g., "I often feel that I put more into our relationship than I get out") on a 5 -point Likert scale $(1=$ strongly agree to $5=$ strongly disagree). The scale includes four reversed items that were positively worded (e.g., "All things considered, my partner and I contribute equally to our relationship"). Higher scores represent a higher perception of relational equity. This scale was translated and adapted into Turkish in a sample of 418 Turkish married individuals (Akçabozan and Hatipoğlu Sümer 2017) and the original one-factor structure was confirmed. Cronbach's alpha value of .87 provided evidence for the reliability of the Turkish RES. In the current study, Cronbach's alpha value was calculated as .80 .

\section{Appreciation in Relationships Scale (AIRS)}

Gordon et al. (2012) developed the AIRS to examine "both the extent to which people feel appreciated by their partners and the extent to which they are appreciative of their partners" (p. 260). The AIRS is comprised of two subscales with 16 items in total $(1=$ strongly disagree to $7=$ strongly agree $)$; nine items for appreciative subscale (e.g., "I often tell my partner how much I appreciate her/him"; "I make sure my partner feels appreciated"), $\alpha=.74$ and 7 items for appreciated subscale (e.g., "My partner often expresses her/his thanks when I do something nice, even if it's really small"; "My partner makes sure I feel appreciated"), $\alpha=.86$. Higher scores indicate higher appreciated and appreciative feelings. This scale was translated and adapted into Turkish in a sample of 416 Turkish married individuals (Akçabozan Kayabol 2017) and the original two-factor structure was confirmed. Cronbach's alpha values of .90 for appreciated and .85 for appreciative subscales showed evidence for the reliability of the Turkish AIRS. ${ }^{1}$ In the current study, Cronbach's Alpha values were .87 for appreciated and .82 for appreciative subscales.

\footnotetext{
${ }^{1}$ More detailed information about the translation and adaptation processes and the results of Exploratory and Confirmatory Factor Analyses of RMBM, RES, and AIRS will be provided by authors upon request.
} 
Relationship Assessment Scale (RAS)

Marital satisfaction was assessed with the Turkish version of RAS (Hendrick 1988), which includes seven items responded on a 5-point Likert scale. Higher scores indicate higher MS. Curun (2001) conducted the Turkish adaptation study of the RAS and reported adequate psychometric properties. Cronbach's alpha coefficient was found to be .86 both in the original and the Turkish adaptation studies. We obtained Cronbach's alpha correlation coefficient of .91 .

\section{Data Collection Procedure}

Before data collection, ethical permission was granted from the University Human Subjects Ethics Committee. Participants were recruited from voluntary individuals who were married for at least one year and living in various cities in Turkey. Data were collected by the first author of this study. Snowball sampling, which is a non-random sampling method, was used in paper-pencil and online surveys. The researcher administered paper-pencil forms along with informed consent forms (including the information about the aim of the study, the total time commitment, risks and discomforts associated with the research, and contact information and affiliation of the researchers) to married individuals who work in various institutions such as universities, birth registration office, hospitals, and private companies. Through the medium of the snowball sampling method, married individuals who could be contacted by the researcher were asked to refer the survey form to other married individual acquaintances who met the criteria. Additionally, the researchers designed an online survey using 'Google Forms' and announced it via e-mail and social media accounts along with the following recruitment statement: "You are invited to participate in a research study which aims to investigate various determinants of marital satisfaction such as relationship maintenance behaviors, equity, and appreciation. You must be above 18 years old, married at least one year, and voluntary to take part in this research. The total time commitment for participation is almost 20 minutes. Your answers will only be used with the purpose of scientific research. You may stop or withdraw your participation at any time." Moreover, when participants clicked the link to participate in the study, they were also asked to declare that they met the inclusion criteria to participate in the study. The individuals who could not be reached in person and/or did not prefer to participate in the paper-pencil survey were also sent the online link. Participants did not receive incentives.

\section{Data Analyses}

We aimed to test two hypothesized structural models. Before conducting Structural Equation Modeling (SEM), we tested preliminary analyses, descriptive statistics, and assumptions of SEM by using SPSS 22 (IBM Corp. Released in, 2013). Constructs in the structural models were identified as composite scores. Using LISREL 8.8 (Jöreskog and Sörbom 1993), we implemented SEM with Maximum Likelihood estimation with robust standard errors and a Satorra-Bentler scaled test statistic (Satorra and Bentler 1994). To interpret the results of SEM, we utilized several fit indices and the suggested cut-off values for each index: $\chi^{2} / d f$-ratio $<5$ (Schumacker and Lomax 2004); CFI, NNFI $\geq .95$ (Hu and Bentler 1999); SRMR < .08 (Browne and Cudeck 1993); RMSEA values smaller than $<0.05$ indicate a close fit, values between 0.05 and 0.10 indicate mediocre fit, and values higher than 0.10 indicate poor fit (Browne and Cudeck 1993).

\section{Results}

\section{Preliminary Analyses}

Prior to conduct SEM, we tested its assumptions (e.g., missing data, normality, influential outliers, linearity and homoscedasticity, and multicollinearity). Considering the amount of missing data for all items was less than $1.4 \%$ and the pattern of missing data was non-significant, we decided to use data imputation through the Expectation-Maximization algorithm (Tabachnick and Fidell 2013). To handle the departure from non-normality, we selected the Satorra-Bentler scalingcorrected test statistic which does not require the assumption of multivariate normality (Satorra and Bentler 1994). Partial regression plots were created and showed no violation of the assumptions of linearity and homoscedasticity. The intercorrelations among the variables were found to be less than the cut-off value of $.90(r=.77$ max. $)$ which satisfied the multicollinearity assumption (Kline 2011).

\section{Descriptive Statistics}

Table 1 shows means, standard deviations, and bivariate correlations. Overall, the means of self-reported use of maintenance behaviors were higher than partner use of maintenance behaviors (e.g., $M=50.99, S D=10.25$ for self-reported openness; $M=48.23, S D=12.24$ for partner openness). All fortyfive correlations among the variables were statistically and positively significant $(p<.01)$. Variables were found correlated varying from small (e.g., between self-reported sharing tasks and MS, $r=.19, p<.01$ ) to large effect (e.g., between partner positivity and MS, $r=.72, p<.01$ ) (Field 2009).

\section{Results of the First Structural Model}

The first structural model tested the direct and indirect associations of the latent variables of self-reported maintenance behaviors (openness, sharing tasks, and positivity), felt 
Table 1 Means, Standard Deviations, and Bivariate Correlations among Study Variables

1 Openness 2 Opennness_P 3 Tasks 4 Tasks_P 5 Positivity 6 Positivity_P 7 Equity 8 Appreciated 9 Appreciative 10 Satisfaction

1.

2. $.70^{* * *}$

3. $.49^{* * *} \quad .35^{* *}$

4. $.44^{* * *} .56^{* *}$

5. $.74^{* * *} \quad .65^{* *}$

6. $.69^{* * *} \quad .77^{* *}$

7. $.45^{* * *} .56^{* *}$

8. $.56^{* *} \quad .72^{* * *}$

9. $.65^{* * *} \quad .57^{* * *}$

10. $.52^{* * *} .59^{* *}$

Range 9-63 9-63

$\begin{array}{lll}M & 50.99 & 48.23\end{array}$

$\begin{array}{lll}S D & 10.25 \quad 12.24\end{array}$

$\begin{array}{llll}.41^{* *} & & & \\ .52^{* *} & .53^{* *} & & \\ .42^{* *} & .63^{* *} & .76^{* *} & \\ .15^{* *} & .53^{* *} & .46^{* *} & .64^{* *} \\ .22^{* *} & .51^{* *} & .56^{* *} & .76^{* *} \\ .33^{* *} & .47^{* *} & .72^{* *} & .68^{* *} \\ .19^{* *} & .56^{* *} & .59^{* *} & .72^{* *} \\ 4-28 & 4-28 & 10-70 & 10-70 \\ 25.25 & 23.78 & 56.71 & 55.04 \\ 3.49 & 4.86 & 9.97 & 11.76\end{array}$

$\begin{array}{ll}.67^{* *} & \\ .56^{* *} & .73^{* *} \\ .67^{* *} & .69^{* *} \\ 10-50 & 7-49 \\ 21.40 & 36.59 \\ 7.48 & 9.60\end{array}$

$\begin{array}{ll}.68^{* *} & \\ 7-63 & 7-35 \\ 48.64 & 29.45 \\ 9.96 & 5.17\end{array}$

Note. $N=602 . * * p<.01$. Openness, Tasks, Positivity represent self-reported, Openness_P, Task_P, Positivity_P represent partner's use of maintenance behaviors

appreciation, and relational equity in explaining MS. Results of this model showed a good fit, $\left[\chi^{2}(1015)=2330.81\right.$, $p=.00 ; \chi^{2} / d f$-ratio $=2.30, \mathrm{CFI}=.98, \mathrm{NNFI}=.98$, SRMR $=.06$, and RMSEA $=0.05(90 \% \mathrm{CI}=.04, .05)$; Browne and Cudeck 1993; Hu and Bentler 1999; Schumacker and Lomax 2004] with significant factor loadings (ranged between .28 and .92). See Fig. 1 for the first hypothesized model. Self-reported maintenance behaviors explained $49 \%$ of the variance in felt appreciation, and $64 \%$ of the variance in equity along with felt appreciation. Overall, all variables explained $73 \%$ of the variance in MS.

Regarding the hypotheses for direct relationships (1st structural model tested $\mathrm{H} 1, \mathrm{H} 3, \mathrm{H} 4$, and $\mathrm{H} 6), 8$ paths out of 12 were found significant. We partially supported Hypothesis 1 and found that the association between MS and positivity was significant and positive $(\beta=.37, p<.01)$; MS and sharing tasks was significant and negative $(\beta=-.14, p<.01)$; MS and openness was not significant $(\beta=-.10, n s$.). Hypotheses
3 and 4 were fully supported and indicated that higher levels of felt appreciation $(\beta=.22, p<.01)$ and equity $(\beta=.50$, $p<.01)$ resulted in greater levels of MS. Additionally, we confirmed Hypothesis $6(\beta=.62, p<.01)$ which shows that individuals perceived greater equity when they felt higher appreciation.

In terms of indirect effects, 10 paths out of 13 were found significant. The indirect relationships between the selfreported use of maintenance behaviors and MS followed two pathways: (1) the individual indirect role of felt appreciation and (2) the serial indirect role of felt appreciation and equity. According to first pathway, the indirect paths from self-reported positivity $(\beta=.11, p<.01)$, sharing tasks $(\beta=$ $-.05, p<.01)$, and openness $(\beta=.08, p<.01)$ to MS were all significant. Specifically, when individuals engaged in openness and positivity more, they felt greater appreciation and experienced higher MS. On the contrary, individuals who reported to engage in sharing tasks more felt lower
Fig. 1 The first hypothesized model with standardized estimates and significant and nonsignificant paths. Only the latent variables were included in the figure. Solid lines indicate significant and dashed lines indicate non-significant paths

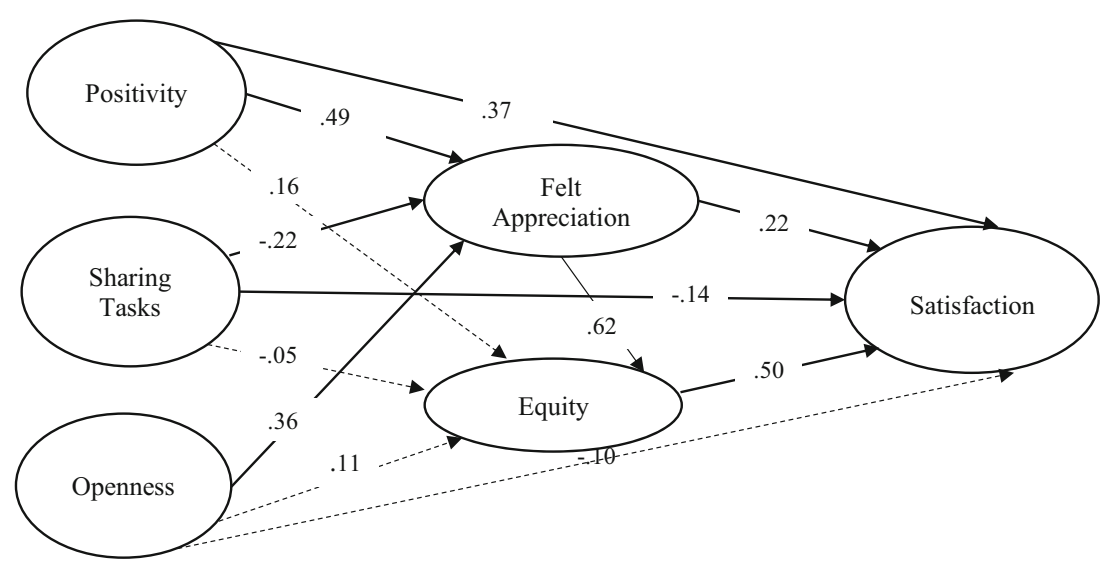


appreciation, and thus experienced lower MS. Moreover, engaging in positivity $(\beta=.30, p<.01)$, sharing tasks $(\beta=-.14$, $p<.01)$, and openness $(\beta=.22, p<.01)$ was significantly associated with equity through felt appreciation. Namely, individuals, who engaged in more openness and positivity and less sharing task behaviors, felt more appreciation and experienced higher equity. All the indirect paths from positivity, sharing tasks, and openness to MS through the indirect role of equity were non-significant $(\beta=.08, n s . ; \beta=-.02, n s$.; $\beta=.05, n s$., respectively).

As a response to RQ1, the second pathway indicated that the indirect paths from each maintenance behavior to MS through the mediators of felt appreciation and equity (in serial) were all significant. Namely, individuals who engaged in more positivity $(\beta=.15, p<.01)$ and openness $(\beta=.11$, $p<.01)$ reported feeling more appreciation and they also perceived higher equity and higher MS in turn. In contrast, individuals who reported to engage in more sharing tasks $(\beta=$ $-.07, p<.01)$ perceived lower levels of appreciation, equity, and MS.

\section{Results of the Second Structural Model}

The second structural model tested the direct and indirect associations of the latent variables of perceived partner maintenance behaviors (openness, sharing tasks, and positivity), relational equity, and appreciative feelings in explaining MS. Results of this model showed a good fit, $\left[\chi^{2}(1018)=\right.$ $2566.01, p=.00 ; \chi 2 / d f$-ratio $=2.32, \mathrm{CFI}=.99, \mathrm{NNFI}=.98$, $\mathrm{SRMR}=.05$, and RMSEA $=0.05(90 \% \mathrm{CI}=.04, .05)$; Browne and Cudeck 1993; Hu and Bentler 1999; Schumacker and Lomax 2004]. All factor loadings were significant and ranged between .13 and .92 . See Fig. 2 for the second hypothesized model. Partners' use of maintenance behaviors explained $67 \%$ of the variance in equity and $64 \%$ of the variance in expressed appreciation along with equity. Overall, all variables explained $75 \%$ of the variance in MS.

Regarding direct paths (2nd structural model tested H2, H5, and H7), 8 paths out of 12 were found significant.
Hypothesis 2 was partially supported and we only found a significant and positive relationship between partner use of positivity and MS $(\beta=.26, p<.01)$ whereas the relationship between MS and partner use of openness was found significant but negative $(\beta=-.12, p<.05)$. The direct path from partner use of sharing tasks to MS was non-significant $(\beta=.01, n s$.$) . We found support for Hypothesis 5$ indicating individuals who had higher levels of appreciative feelings also experienced higher MS $(\beta=.33, p<.01)$. Furthermore, as hypothesized (Hypothesis 7), the direct path from perceived equity to expressed appreciation was also found positive and significant $(\beta=.20, p<.05)$.

In terms of indirect effects, 8 indirect paths out of 13 were found significant. The indirect relationships between the partner use of maintenance behaviors and MS followed three pathways: individual indirect roles of (1) equity and (2) expressed appreciation and (3) the serial indirect role of equity and appreciative feelings. According to the first pathway, results indicated that higher levels of partner use of positivity $(\beta=.27, p<.01)$ and sharing tasks $(\beta=.08, p<.01)$ were related to higher levels of MS through the indirect role of equity. This indirect role was not significant for the relationship between partner openness and MS $(\beta=.04, n s$.). Additionally, having higher perceptions of partner use of positivity $(\beta=.12$, $p<.05)$ and sharing tasks $(\beta=.04, n s$. $)$ were associated with expressed appreciation through the indirect role of equity. This indirect role was again not significant for partner use of openness $(\beta=.02, n s$.). The second pathway showed that the indirect role of expressed appreciation was significant in explaining the relationship between partner use of positivity $(\beta=.24, p<.01)$ and MS as well as equity and MS $(\beta=.06$, $p<.05)$. Namely, when individuals perceived higher use of positivity from their partners and higher equity in their marriages, they also reported expressing higher appreciation and experienced higher MS. However, the indirect role of expressed appreciation was not significant in explaining the role of partner use of openness and sharing tasks on MS $(\beta=$ $-.03, n s . ; \beta=-.01, n s$., respectively). Finally, the third pathway also showed a similar pattern indicating that individuals
Fig. 2 The second hypothesized model with standardized estimates and significant and nonsignificant paths. Only the latent variables were included in the figure. Solid lines indicate significant and dashed lines indicate non-significant paths

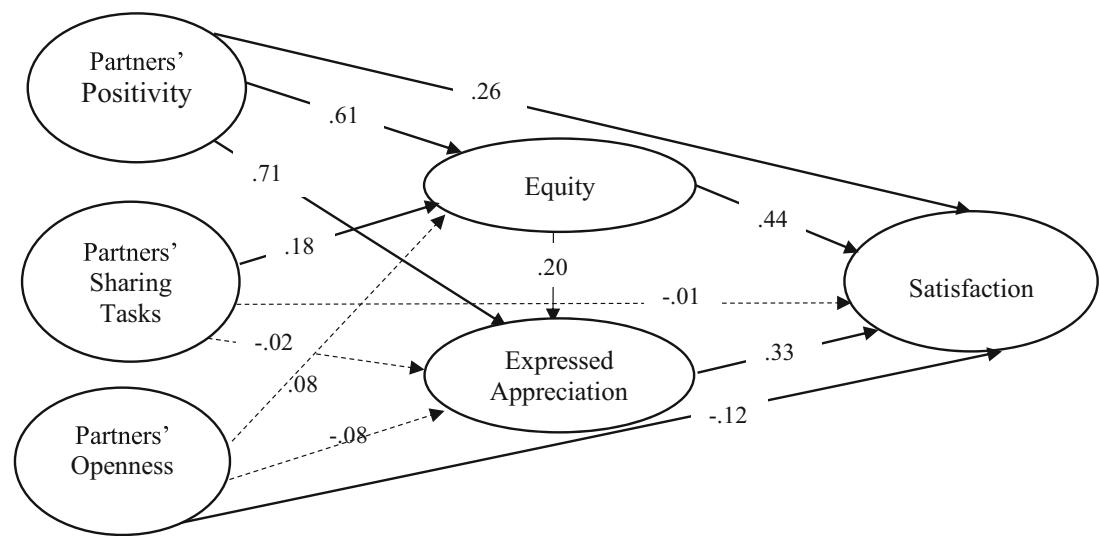


with higher partner use of positivity $(\beta=.04, p<.01)$ and sharing tasks behaviors $(\beta=.01, p<.05)$ reported to perceive more equity, express more appreciation, and accordingly experience higher MS. This indirect role was not significant for the relationship between partner use of openness and MS $(\beta=.01, n s$.$) .$

\section{Discussion}

We utilized the perspective of equity theory to examine how self-reported and partner use of maintenance behaviors predict MS through individual and serial indirect roles of perceived equity and felt and expressed appreciation. Although the variables tested in each model varied across two models (selfreported maintenance behaviors, perceived equity, and felt appreciation in the first model and partners' use of maintenance behaviors, perceived equity, and expressed appreciation in the second model), we discussed the findings of each model together and made comparative discussions when possible; yet, we also mentioned unique findings of each model.

\section{Discussion of the Direct Relationships}

Hypotheses 1 and 2 proposed that higher self-reported and partner use of each maintenance behavior is associated with higher MS. Results regarding positivity behavior, as expected, showed that self-reported and partner engagement in positivity were associated with higher levels of MS. Similarly, there is prolific evidence in the literature supporting the primary predictor role of self-reported and partner use of positivity on MS, including studies from various cultural contexts (e.g., Dainton et al. 1994; Johnson 2009; Rehman and Holtzworth-Munroe 2007; Stafford and Canary 1991). Results of the current study also echo the findings of the only study in which maintenance behaviors were examined in a sample from Turkey and positivity was found a positive predictor of MS (Torun 2005). Our results, indicating a negative association between self-reported sharing task and MS, support the notion that task sharing requires routine engagement (Dainton and Aylor 2002) which may lead to the perception that a partner's task sharing functions as a cost to them, subsequently resulting in lower satisfaction. On the other hand, this result is not consistent with the previous research indicating a significant and positive association between one's use of task sharing and MS (e.g., Johnson 2009; Stafford and Canary 1991; Torun 2005).

The nonsignificant association between partner use of sharing tasks and MS in this study was in line with the previous research (e.g., Dainton 2000; Stafford 2011). Still, our finding is not consistent with the studies providing evidence for the significant and positive predictor role of task sharing on MS (e.g., Ballard-Reisch et al. 1999; Dainton et al. 1994; Torun
2005). A possible explanation for this could be that individuals might overlook or be less aware of their partners' task sharing because it is a routine, less communicative, and highly expected behavior (Dainton 2000; Johnson 2009). Furthermore, individuals' satisfaction could be promoted depending on whether their expectations are met regarding task sharing rather than actual levels of their partners' task sharing performance.

Moreover, the non-significant direct path from selfreported openness to MS was consistent with the studies indicating a less influential role of openness on satisfaction compared to other behaviors (Johnson 2009; Stafford and Canary 1991; Torun 2005) whereas inconsistent with the other studies providing evidence for a significant predictor role of openness on MS (e.g., Lee 2006; Stafford et al. 2000). For partner use of openness, our results support previous research that have demonstrated a significant but negative relationship between perceived openness from a partner and MS (e.g., Dainton et al. 1994; Stafford and Canary 1991; Stafford 2011). Openness allows partners to reveal their feelings and thoughts about themselves and their relationships, which can be considered as beneficial for the quality of the relationship. Conversely, it is also possible that one partner's disclosure about him/herself or the nature of the relationship can cause stress for the other partner. Accordingly, social exchange theory also proposes that openness has both a positive and negative effect for partners who are disclosing themselves and who are exposed to the choice to disclose certain information by their partners (Hendrick 1981). It was also proposed that the effect of selfopenness may lose its importance in long-term marriages and decrease at the later stages of the marriage (Hendrick 1981; Rosenfeld and Bowen 1991). Considering our sample with an average marital length of almost 10 years, we argue that being open about feelings, thoughts, and progress of marriage might not be as influential as for newlyweds and might even have an aversive effect on partners' MS.

We have fully supported Hypotheses 3-5 (Model 1 and 2) and showed that individuals were highly satisfied when they perceived higher equity and felt and expressed higher appreciation. The positive and strong direct relationship between equity and MS is in line with the theoretical implications of equity theory and the findings of numerous empirical studies (e.g., Stafford and Canary 2006, Weigel et al. 2006). The transition of Turkey from a patriarchal to an egalitarian society in recent decades (Sunar and Fișek 2005) could also be supportive of the positive influence of perceived equity on MS in a sample of Turkish married individuals. Further, positive direct paths from felt and expressed appreciation to MS were also as expected given the results of past research (Algoe et al. 2010; Gordon et al. 2012). Indeed, felt and expressed appreciation should remind individuals that their partner's awareness of his/her value likely contributes to an increase in their MS. 
Finally, we fully confirmed our Hypotheses $6-7$. We verified and built upon the existing literature by revealing the positive and significant association between felt appreciation and equity (e.g., Hawkins et al. 1998). Additionally, although no prior research demonstrated the association between equity and expressed appreciation, based on the principles of equity theory and the results of our study, we can confidently argue that perceived equity should stimulate individuals' positive perceptions and elicit more appreciation of their partners.

\section{Discussion of Indirect Relationships}

Starting from the first model, significant indirect relationships occurred through (1) the individual indirect role of felt appreciation and (2) felt appreciation and perceived equity in serial (which resulted from felt appreciation). Results of the present study have expanded upon the existing maintenance literature by illustrating that the self-reported maintenance behaviors do not have a direct role in perceived equity, and perceived equity only has an indirect role when individuals reported felt appreciation from their spouses. This result has offered insight into the protective role of felt appreciation in terms of promoting increases in perceived equity and MS when individuals engage in maintenance behaviors. In the second model, we found that the relationships between partner use of positivity and MS as well as perceived task sharing and MS were significant but the relationship between partner use of openness and MS was not significantly mediated. This is true both through the (1) individual indirect role of perceived equity and (2) the indirect role of mediators in serial (expressed appreciation resulted from perceived equity).

Starting from positivity behaviors, we validated and extended previous research in which positivity appears to be one of the most powerful strategies to ensure relationship satisfaction (e.g., Canary and Stafford 1992; Gordon and Baucom 2009) with the inclusion of evidence for the indirect roles of equity and appreciation. Individuals who enacted positivity behaviors might perceive their partners to be receiving more benefits in the relationship compared to themselves (Kollock et al. 1994). Yet, higher felt appreciation might help them to overcome their doubts and be motivated to keep the relationship in an equitable and satisfying state while they are engaging in higher positivity behaviors. Similarly, partner use of positivity behaviors may arouse the benefactors' appreciative feelings as a reminder to celebrate their partner's worth. This would, in turn, promote their satisfaction. Moreover, this study contributed to the limited existing research which previously had not provided evidence for the predictor role of appreciative feelings on partner positivity (Lambert and Fincham 2011). Our results have confirmed the premise that being positive is crucial and engagement in positive emotions encourages people to feel more confident and content in their marriages (Prager and Roberts 2004; Williamson et al. 2013).
Consistent with the previous studies, our findings regarding the self-reported task sharing showed that a higher level of felt appreciation is a motivator of greater satisfaction under the conditions of the use of relationally costly behaviors such as family chores (e.g., Berger and Janoff-Bulman 2006; Berger 2000). Moreover, this result also confirms the negative mediator role of perceived appreciation in explaining the relationship between costly maintenance behaviors (sacrifices) and relationship satisfaction (Young and Curran 2016). Previous research presented that partners perceived less equity when they did not feel appreciated for their engagement in task sharing, and the negative influence of inequity on satisfaction disappeared when partners perceive themselves as highly appreciated (Hawkins et al. 1998). Considering the more routine and less exciting nature of task and responsibility sharing, partners might be perceiving their engagement in task sharing as highly costly investments that associated with lower levels of MS. As such, the indirect role of felt appreciation promotes a sense of equity as well as MS. In comparison, indicating the positive indirect role of the perceived equity and expressed appreciation is complementary to the findings of the first model. Although the previous literature has not yet examined the role of expressed appreciation with task sharing, one could expect that partner engagement in tasks would contribute to individuals' appreciative feelings towards their partners and perceived levels of MS. Nevertheless, either the direct relationships between partner use of task sharing with appreciative feelings as well as MS or the individual indirect role of appreciative feelings was not significant. Yet, with the addition of the perceived equity, the serial mediator role turned into being significant and positive. This is parallel with the previous support for the protective role of contentment and perceived equity of household labor and family work on the relationship between partners' actual task sharing and MS (Lavee and Katz 2002). Together, the significant and positive serial mediation provided coherent and supplementary evidence for previous research which indicated that perceived higher partner investment was associated with greater relationship commitment through the mediator role of expressed gratitude toward a romantic partner (Joel et al. 2013).

Regarding the results for openness behavior, we found that the non-significant direct relationship between self-reported openness and MS became significant through the indirect role of felt appreciation and perceived equity. We argue that individuals might perceive enactment in voicing relational concerns and self-disclosure about themselves as relationally costly; and therefore, expect their openness to being cared about and repaid by their partners. A previous study, in which the expression of gratitude was found to be related to an increase in voicing relational concerns (akin to openness) about a romantic partner or friend is aligned with our conclusions (Lambert and Fincham 2011). For partner use of openness, it can be expected that perceived equity and appreciative 
feelings might buffer the negative direct role of openness on MS; however, the non-significant results for indirect effects did not confirm this expectation. Consistent with the premise that perceived gratitude could be less when the benefactors were intimately close to the beneficiaries and expected to enact relational benefits for each other (McCullough et al. 2001), individuals might believe that their partners are naturally obligated to disclose themselves and might not consider their openness as a relational benefit to be exchanged in the evaluation of equity. Furthermore, the significant and strong role of partner use of positivity towards MS could also mitigate the influential role of partner openness. Consequently, in understanding MS, we found that the indirect roles follow varying paths and even if an exchange of resources is inevitable in marriages, perceived equity is influenced by felt appreciation when individuals report their self-engagements, and perceived equity is influential on individuals' expressed appreciation when they report their perceptions of partner engagement.

\section{Implications for Theory, Research, and Practice}

Considering that principles of equity theory are expected and mostly ensured to be applied to relationship maintenance in cultures with more modern and Westernized values, our results are noteworthy in terms of demonstrating initial evidence for the significant associations between perceived equity, use of maintenance behaviors, and MS in a married sample from Turkey. In other words, even though we did not seek to compare different cultures in this study, we revealed that the equity-maintenance connection is also relevant to marital partners from a culture holding less individualistic, modern, and secular but more collectivist and traditional values compared to Western culture. We hope this will encourage scholars to examine how equity theory functions and relationship maintenance patterns in various samples representing different cultural modernization values both alike with and different from Turkey. Secondly, the application of equity theory has moved beyond the premise that people are more likely to engage in maintenance behaviors when they feel equitable, instead, we provided evidence that relational equity can also function as an outcome and mediator in understanding the association between the use of maintenance behaviors and MS. Thus, future studies should consider testing the application of equity theory principles by paying attention to potential bidirectionality between the use of maintenance behaviors and relational equity.

To our knowledge, this study is the first attempt to investigate appreciation in a sample of married individuals from Turkey. Our results have also built on the theoretical framework and growing body of research on appreciation/gratitude in intimate relationships by highlighting the protective role of both felt and expressed appreciation for married partners. Our findings challenge researchers to pay closer attention to examining the utility of appreciation in relationship maintenance and contentment. Overall, as is evident from the recent literature review on relationship maintenance (Ogolsky et al. 2017), we have provided another perspective of relationship maintenance research by integrating individual and interactive processes (i.e., perceived equity and appreciation) to better understand how self- and partner-reported maintenance behaviors explain MS. There are countless directions future research could take towards enhancing our understanding of the role of equity theory perspective and appreciation both in married participant samples and in other close relationship types where maintenance matters, such as dating (e.g., Yum and Li 2007) and friendships (e.g., Sanchez et al. 2018).

The implications of our results are significant for practitioners, as well. Marriage and relationship education programs, aiming to help individuals reduce relational distress and maintain satisfying relationships could benefit from our conclusions (Blanchard et al. 2009). Although engaging in maintenance behaviors might be considered functional for MS, contradictory findings of our study (i.e., the nonsignificant or negative role of openness on MS) could encourage researchers to reconsider the design of the scope and practice of established interventions. On the other hand, in marital intervention and prevention programming, the benefits of positive interaction with the partner, sense of equity, and spousal appreciation, both for the beneficiaries and expressers, could be reinforced. In their counseling sessions, practitioners can assist couples who are suffering from the problems in the allocation of household tasks by advocating discussions about their expectations of task sharing, how their perceptions of equity in terms of sharing responsibilities could be met, and what appreciation from their partners means for them. This way, an individual's engagement in task sharing might be revised to avoid undesired effects in their experiences of MS. Above all, professionals should instill the idea that relationships are not static entities, but rather, they require effort to ensure successful maintenance. Both partners of the relationship should work to gain awareness in terms of how to keep their marriages at a desired level of satisfaction.

\section{Limitations and Recommendations for Further Research}

Our study is not without its limitations and we have identified possible directions for future research. First, our sample consists mostly of long-term married individuals from urban areas, which ultimately represents a profile of highly educated people. Thus, future researchers could replicate the present study by collecting data better representing a variety in marital length. Collecting data from rural areas would also enrich the results of the current study considering that social and economic disadvantages have an influence in explaining the associations between partners' behaviors (e.g., communication 
patterns and positivity and negativity) and marital satisfaction (Ross et al. 2019; Williamson et al. 2013). Moreover, considering the fact that certain maintenance behaviors may be more useful than others in different relationship types, the investigation and comparison of different romantic relationships (e.g., dating, cohabiting, long-distance, same-sex partners) would also substantiate the findings of our study.

Second, we collected data from one partner of a dyad and we did not infer causality among study variables. However, the recruitment of dyadic data and investigate causal relationships through longitudinal, daily diary, and experimental studies would address the missing data regarding causality and bidirectionality among the study variables (Gordon et al. 2012). One final modification that could be made concerns how conditions of equity and appreciation relate to maintenance and MS. For instance, we did not examine the inequity conditions of under- and over-benefitedness; however, perceived (in)equity (over-benefitedness, equity, and underbenefitedness) is more likely to be in curvilinear association with relationship maintenance and MS (Stafford and Canary 2006), which could result in complexity in the mediator role of perceived (in)equity.

Lastly, because we did not include the potential demographic variables as controls or moderators into our models, we encourage the researchers to explore whether the structural models in the current study vary by demographics (e.g., gender, marital length).

On behalf of all authors, the corresponding author states that there is no conflict of interest.

Data Availability Statement The data that support the findings of this study are available from the corresponding author, [N.K], upon reasonable request.

\section{References}

Akçabozan Kayabol, N. B. (2017). Relationship maintenance behaviors and marital satisfaction: The mediator roles of relational equity and appreciation (unpublished dissertation). Ankara: Middle East Technical University.

Akçabozan, N. B., \& Hatipoğlu Sümer, Z. (2017). Turkish adaptation of the relational equity scale: Validity and reliability studies. Proceedings of the $26^{\text {th }}$ International Conference on Educational Sciences, 355-362. https://doi.org/10.14527/9786053187974.

Akın, A., \& Yalnız, A. (2015). Turkish version of expression of gratitude in relationships measure (EGRM): The study of validity and reliability. The Journal of International Social Research, 8(37), 539-544.

Algoe, S. B., Gable, S. L., \& Maisel, N. C. (2010). It's the little things: Everyday gratitude as a booster shot for romantic relationships. Personal Relationships, 17(2), 217-233. https://doi.org/10.1111/j. 1475-6811.2010.01273.x.

Ballard-Reisch, D. S., Weigel, D. J., \& Zaguidoulline, M. G. (1999). Relational maintenance behaviors, marital satisfaction, and commitment in Tatar, Russian, and mixed Russian-Tatar marriages: An exploratory analysis. Journal of Family Issues, 20(5), 677-697. https://doi.org/10.1177/019251399020005006.

Barton, A. W., Futris, T. G., \& Nielsen, R. B. (2015). Linking financial distress to marital quality: The intermediary roles of demand/ withdraw and spousal gratitude expressions. Personal Relationships, 22(3), 536-549.

Bello, R. S., Brandau-Brown, F. E., Zhang, S., \& Ragsdale, J. D. (2010). Verbal and nonverbal methods for expressing appreciation in friendships and romantic relationships: A cross-cultural comparison. International Journal of Intercultural Relations, 34(3), 294-302. https://doi.org/10.1016/j.ijintrel.2010.02.007.

Berger, A. R. (2000). The role of appreciation in close relationships (unpublished doctoral dissertation). Ann Arbor: University of Massachusetts Amherst.

Berger, A. R., \& Janoff-Bulman, R. (2006). Costs and satisfaction in close relationships: The role of loss-gain framing. Personal Relationships, 13(1), 53-68. https://doi.org/10.1111/j.1475-6811. 2006.00104.x.

Blanchard, V. L., Hawkins, A. J., Baldwin, S. A., \& Fawcett, E. B. (2009). Investigating the effects of marriage and relationship education on couples' communication skills: A meta-analytic study. Journal of Family Psychology, 23(2), 203-214. https://doi.org/10. 1037/a0015211.

Browne, M. W., \& Cudeck, R. (1993). Alternative ways of assessing model fit. In K. A. Bollen \& J. S. Long (Eds.), Testing structural equation models (pp. 136-162). Newbury Park: Sage.

Canary, D. J., \& Stafford, L. (1992). Relational maintenance strategies and equity in marriage. Communication Monographs, 59(3), 243267. https://doi.org/10.1080/03637759209376268.

Canary, D. J., \& Stafford, L. (2001). Equity in preservation of personal relationships. In J. H. Harvey, \& A. Wenzel (Eds.), Close romantic relationships: Maintenance and enhancement (pp. 133-151). Mahwah, NJ: Lawrence Erlbaum Associates.

Canary, D. J., Stafford, L., Hause, K. S., \& Wallace, L. A. (1993). An inductive analysis of relational maintenance strategies: Comparisons among lovers, relatives, friends, and others. Communication Research Reports, 10, 3-14. https://doi.org/10.1080/ 08824099309359913.

Canary, D. J., \& Stafford, L. (1994). Maintaining relationships through strategic and routine interaction. In D. J. Canary \& L. Stafford (Eds.), Communication and relational maintenance (pp. 3-22). San Diego: Academic Press.

Costello, A. B., \& Osborne, J. W. (2005). Best practices in exploratory factor analysis: Four recommendations for getting the most from your analysis. Practical Assessment, Research \& Evaluation, 10(7), 1-9.

Curun, F. (2001). The effects of sexism and sex role orientation on romantic relationship satisfaction (unpublished master's thesis). Ankara: Middle East Technical University.

Dainton, M. (1994). An examination of routine and strategic interactions in maintained marital relationships (unpublished doctoral dissertation). Ohio: The Ohio State University.

Dainton, M. (2000). Maintenance behaviors, expectations for maintenance, and satisfaction: Linking comparison levels to relational maintenance strategies. Journal of Social and Personal Relationships, 17(6), 827-842. https://doi.org/10.1177/ 0265407500176007.

Dainton, M. (2016). Equity, equality, and self-interest in marital maintenance. Communication Quarterly, 65, 1-21. https://doi.org/10. 1080/01463373.2016.1227346.

Dainton, M., \& Aylor, B. (2002). Routine and strategic maintenance efforts: Behavioral patterns, variations associated with relational length, and the prediction of relational characteristics. Communication Monographs, 69(1), 52-66. https://doi.org/10. 1080/03637750216533. 
Dainton, M., Stafford, L., \& Canary, D. J. (1994). Maintenance strategies and physical affection as predictors of love, liking, and satisfaction in marriage. Communication Reports, 7(2), 88-98. https://doi.org/ 10.1080/08934219409367591.

Dainton, M., \& Zelley, E. D. (2006). Social exchange theories: Interdependence and equity. In D. O. Braithwaite \& L. A. Baxter (Eds.), Engaging theories in family communication: Multiple perspectives (pp. 243-259). Thousand Oaks: Sage Publications.

Duck, S. (1988). Relating to others. Chicago: Dorsey Press.

Field, A. (2009). Discovering statistics using SPSS (3rd ed.). Thousand Oaks, CA: Sage.

Fincham, F. D., Rogge, R., \& Beach, S. R. H. (2018). Relationship satisfaction. In A. L. Vangelisti \& D. Perlman (Eds.), The Cambridge handbook of personal relationships (p. 422-436). Cambridge University Press. https://doi.org/10.1017/9781316417867.033.

Floyd, F. J., \& Wasner, G. H. (1994). Social exchange, equity, and commitment Structural equation modeling of dating relationships. Journal of Family. Psychology, 8(1), 55-73. https://doi.org/10. 1037/0893-3200.8.1.55.

Gordon, A. M., Impett, E. A., Kogan, A., Oveis, C., \& Keltner, D. (2012). To have and to hold: Gratitude promotes relationship maintenance in intimate bonds. Journal of Personality and Social Psychology, 103(2), 257-274. https://doi.org/10.1037/a0028723.

Gordon, C. L., \& Baucom, D. H. (2009). Examining the individual within marriage: Personal strengths and relationship satisfaction. Personal Relationships, 16(3), 421-435. https://doi.org/10.1111/j.1475-6811. 2009.01231.x.

Gurbuz, S., Costigan, R., \& Teke, K. (2018). Does being positive work in a Mediterranean collectivist culture? Relationship of core selfevaluations to job satisfaction, life satisfaction, and commitment. Current Psychology, 1-16. https://doi.org/10.1007/s12144-0189923-6.

Hatfield, E., \& Rapson, R. L. (2011). Equity theory in close relationships. In P. A. M. Van Lange, A. W. Kruglanski, \& E. T. Higgins (Eds.), Handbook of theories of social psychology (pp. 200-217). London: Glyph International.

Hatfield, E., Traupmann, J., Sprecher, S., Utne, M., \& Hay, J. (1985). Equity and intimate relations: Recent research. In D. W. Ickes (Ed.), Compatible and incompatible relationships (pp. 91-117). New York: Springer-Verlag.

Hawkins, A. J., Marshall, C. M., \& Allen, S. M. (1998). The orientation toward domestic labor questionnaire: Exploring dual-earner wives' sense of fairness about family work. Journal of Family Psychology, 12(2), 244-258. https://doi.org/10.1037/0893-3200.12.2.244.

Hendrick, S. S. (1981). Self-disclosure and marital satisfaction. Journal of Personality and Social Psychology, 40(6), 1150-1159. https://doi. org/10.1037/0022-3514.40.6.1150.

Hendrick, S. S. (1988). A generic measure of relationship satisfaction. Journal of Marriage and Family, 50(1), 93-98. https://doi.org/10. 2307/352430.

Hu, L., \& Bentler, P. M. (1999). Cutoff criteria for fit indexes in covariance structure analysis: Conventional criteria versus new alternatives. Structural Equation Modeling: A Multidisciplinary Journal, 6(1), 1-55. https://doi.org/10.1080/10705519909540118.

IBM Corp. Released in. (2013). IBM SPSS statistics for windows, version 22.0. Armonk: IBM Corp.

Inglehart, R., \& Welzel, C. (2005). Modernization, cultural change, and democracy: The human development sequence. New York: Cambridge University Press.

Jackson, D. (2010). Equity, maintenance behaviors, and relational satisfaction in distressed partners (unpublished doctoral dissertation). Arizona: Arizona State University.

Joel, S., Gordon, A. M., Impett, E. A., MacDonald, G., \& Keltner, D. (2013). The things you do for me perceptions of a romantic partner's investments promote gratitude and commitment. Personality and
Social Psychology Bulletin, 39(10), 1333-1345. https://doi.org/10. 1177/0146167213497801.

Johnson, S. L. (2009). Individual and interdependent analyses of relational maintenance, sexual communication, and marital quality (unpublished doctoral dissertation). Arizona: Arizona State University.

Jöreskog, K. G., \& Sörbom, D. (1993). LISREL 8: Structural equation modeling with the SIMPLIS command language. Chicago: Scientific Software International.

Kagitcibasi, C. (1997). Individualism and collectivism. In J. W. Berry, M. H. Segall, \& C. Kagitcibasi (Eds.), Handbook of cross-cultural psychology, Social behavior and applications (Vol. 3, pp. 1-49). Boston: Allyn \& Bacon.

Kline, R. B. (2011). Principles and practice of structural equation modeling. New York: Guilford Press.

Kollock, P., Blumstein, P., \& Schwartz, P. (1994). The judgment of equity in intimate relationships. Social Psychology Quarterly, 57(4), 340-351. https://doi.org/10.2307/2787160.

Kubacka, K. E., Finkenauer, C., Rusbult, C. E., \& Keijsers, L. (2011). Maintaining close relationships: Gratitude as a motivator and a detector of maintenance behavior. Personality and Social Psychology Bulletin, 37(10), 1362-1375. https://doi.org/10.1177/ 0146167211412196

Lambert, N. M., \& Fincham, F. D. (2011). Expressing gratitude to a partner leads to more relationship maintenance behavior. Emotion, 11(1), 52-60. https://doi.org/10.1037/a0021557.

Lavee, Y., \& Katz, R. (2002). Division of labor, perceived fairness, and marital quality: The effect of gender ideology. Journal of Marriage and Family, 64(1), 27-39. https://doi.org/10.2307/2580391.

Lee, E. (2006). Relationship maintenance behaviors in marital couples (unpublished doctoral dissertation). Claremont: Claremont Graduate University.

McCullough, M. E., Kilpatrick, S. D., Emmons, R. A., \& Larson, D. B. (2001). Is gratitude a moral affect? Psychological Bulletin, 127(2), 249-266. https://doi.org/10.1037/0033-2909.127.2.249.

Ogolsky, B. G., Monk, J. K., Rice, T. M., Theisen, J. C., \& Maniotes, C. R. (2017). Relationship maintenance: A review of research on romantic relationships. Journal of Family Theory and Review, 9, 275306. https://doi.org/10.1111/jftr.12205.

Ogolsky, B. G., \& Bowers, J. R. (2013). A meta-analytic review of relationship maintenance and its correlates. Journal of Social and Personal Relationships, 30(3), 343-367. https://doi.org/10.1177/ 0265407512463338 .

Powell, L. A. (2005). Justice judgments as complex psychocultural constructions: An equity-based heuristic for mapping two- and threedimensional fairness representations in perceptual space. Journal of Cross-Cultural Psychology, 36(1), 48-73. https://doi.org/10.1177/ 0022022104271426 .

Prager, K. J., \& Roberts, L. J. (2004). Deep intimate connection: Self and intimacy in couple relationships. In D. J. Mashek \& A. Aron (Eds.), Handbook of closeness and intimacy (pp. 43-60). Mahwah: Lawrence Erlbaum Associates.

Rehman, U. S., \& Holtzworth-Munroe, A. (2007). A cross-cultural examination of the relation of marital communication behavior to marital satisfaction. Journal of Family Psychology, 21(4), 759-763. https://doi.org/10.1037/0893-3200.21.

Rosenfeld, L. B., \& Bowen, G. L. (1991). Marital disclosure and marital satisfaction: Direct effect versus interaction-effect models. Western Journal of Speech Communication, 55(1), 69-84. https://doi.org/10. 1080/10570319109374371.

Ross, J. M., Karney, B. R., Nguyen, T. P., \& Bradbury, T. N. (2019). Communication that is maladaptive for middle-class couples is adaptive for socioeconomically disadvantaged couples. Journal of Personality and Social Psychology, 116(4), 582-597. https://doi. org/10.1037/pspi0000158 
Sabatelli, R. M., \& Cecil-Pigo, E. F. (1985). Relational interdependence and commitment in marriage. Journal of Marriage and Family, 47(4), 931-937. https://doi.org/10.2307/352336.

Sanchez, M., Haynes, A., Parada, J, C., \& Demir, M. (2018). Friendship maintenance mediates the relationship between compassion for others and happiness. Current Psychology, https://doi.org/10.1007/ s12144-017-9779-1

Satıcı, S. A., Uysal, R., \& Akın, A. (2014). Forgiveness and vengeance: The mediating role of gratitude. Psychological Reports, 114(1), 157-168. https://doi.org/10.2466/07.09.PR0.114k11w9.

Satorra, A., \& Bentler, P. M. (1994). Corrections to test statistics and standard errors on covariance structure analysis. In A. Von Eye \& C. C. Clogg (Eds.), Latent variables analysis: Applications for developmental research (pp. 399-419). Thousand Oaks: Sage.

Schramm, D. G., Marshall, J. P., Harris, V. W., \& Lee, T. R. (2005). After "I do": The newlywed transition. Marriage \& Family Review, 38(1), 45-67. https://doi.org/10.1300/J002v38n01_05.

Schumacker, R. E., \& Lomax, R. G. (2004). A beginner's guide to structural equation modeling (2nd ed.). Mahwah: Lawrence Erlbaum Associates.

Sprecher, S. (1992). Social exchange perspectives on the dissolution of close relationships. In T. L. Orbuch (Ed.), Close relationship loss (pp. 47-66). New York: Springer-Verlag.

Sprecher, S., \& Schwartz, P. (1994). Equity and balance in the exchange of contributions in close relationships. In M. J. Lerner \& G. Mikula (Eds.), Entitlement and the affectional bond (pp. 11-41). New York: Plenum.

Stafford, L. (2003). Maintaining romantic relationships: Summary and analysis of one research program. In D. J. Canary \& M. Dainton (Eds.), Maintaining relationships through communication: Relational, contextual, and cultural variations (pp. 51-77). Mahwah: Lawrence Erlbaum Associates.

Stafford, L. (2011). Measuring relationship maintenance behaviors: Critique and development of the revised relationship maintenance behavior scale. Journal of Social and Personal Relationships, 28(2), 278-303. https://doi.org/10.1177/0265407510378125.

Stafford, L., \& Canary, D. J. (1991). Maintenance strategies and romantic relationship type, gender, and relational characteristics. Journal of Social and Personal Relationships, 8(2), 217-242. https://doi.org/ 10.1177/0265407591082004.

Stafford, L., \& Canary, D. J. (2006). Equity and interdependence as predictors of relational maintenance strategies. Journal of Family Communication, 6(4), 227-254. https://doi.org/10.1207/ s15327698jfc0604 1.

Stafford, L., Dainton, M., \& Haas, S. (2000). Measuring routine and strategic relational maintenance: Scale revision, sex versus gender roles, and the prediction of relational characteristics.
Communication Monographs, 67(3), 306-323. https://doi.org/10. 1080/03637750009376512.

Sunar, D., \& Fișek, G. O. (2005). Contemporary Turkish families. In J. L. Roopnarine \& U. P. Gielen (Eds.), Families in global perspective (pp. 169-183). Boston: Allyn and Bacon.

Tabachnick, B, G., \& Fidell, L, S. (2013). Using multivariate statistics. (6th ed.) Boston: Pearson/Allyn \& Bacon.

Thibaut, J. W., \& Kelley, H. H. (1959). The social psychology of groups. New York: Wiley.

Torun, C. (2005). Relationship Maintenance Strategies and Marital Satisfaction among Turkish Couples (Unpublished master's thesis). İstanbul: Boğaziçi University.

Young, V. J., \& Curran, M. A. (2016). Intimacy behaviors and relationship satisfaction for cohabitors: Intimate sacrifices are not always beneficial. The Journal of Psychology, 150(6), 779-792. https://doi. org/10.1080/00223980.2016.1187110.

Yum, Y., \& Canary, D. J. (2009). Cultural differences in equity theory predictions of relational maintenance strategies. Human Communication Research, 35(3), 384-406. https://doi.org/10. 1111/j.1468-2958.2009.01356.x.

Yum, Y., \& Li, H. Z. (2007). Associations among attachment style, maintenance strategies, and relational quality across cultures. Journal of Intercultural Communication Research, 36(2), 71-89. https://doi.org/10.1080/17475750701478612.

Ward, P. J., Lundberg, N. R., Zabriskie, R. B., \& Berrett, K. (2009). Measuring marital satisfaction: A comparison of the revised dyadic adjustment scale and the satisfaction with married life scale. Marriage \& Family Review, 45(4), 412-429.

Weigel, D. J., Bennett, K. K., \& Ballard-Reisch, D. S. (2006). Influence strategies in marriage: Self and partner links between equity, strategy use, and marital satisfaction and commitment. Journal of Family Communication, 6(1), 77-95. https://doi.org/10.1207/ s15327698jfc0601 5 .

Williamson, H. C., Karney, B. R., \& Bradbury, T. N. (2013). Financial strain and stressful events predict newlyweds' negative communication independent of relationship satisfaction. Journal of Family Psychology, 27(1), 65-75. https://doi.org/10.1037/a0031104.

Yum, Y., Canary, D. J., \& Baptist, J. (2015). The roles of culture and fairness in maintaining relationships: A comparison of romantic partners from Malaysia, Singapore, and the United States. International Journal of Intercultural Relations, 44, 100-112. https://doi.org/10.1016/j.ijintrel.2014.12.003

Publisher's Note Springer Nature remains neutral with regard to jurisdictional claims in published maps and institutional affiliations. 